
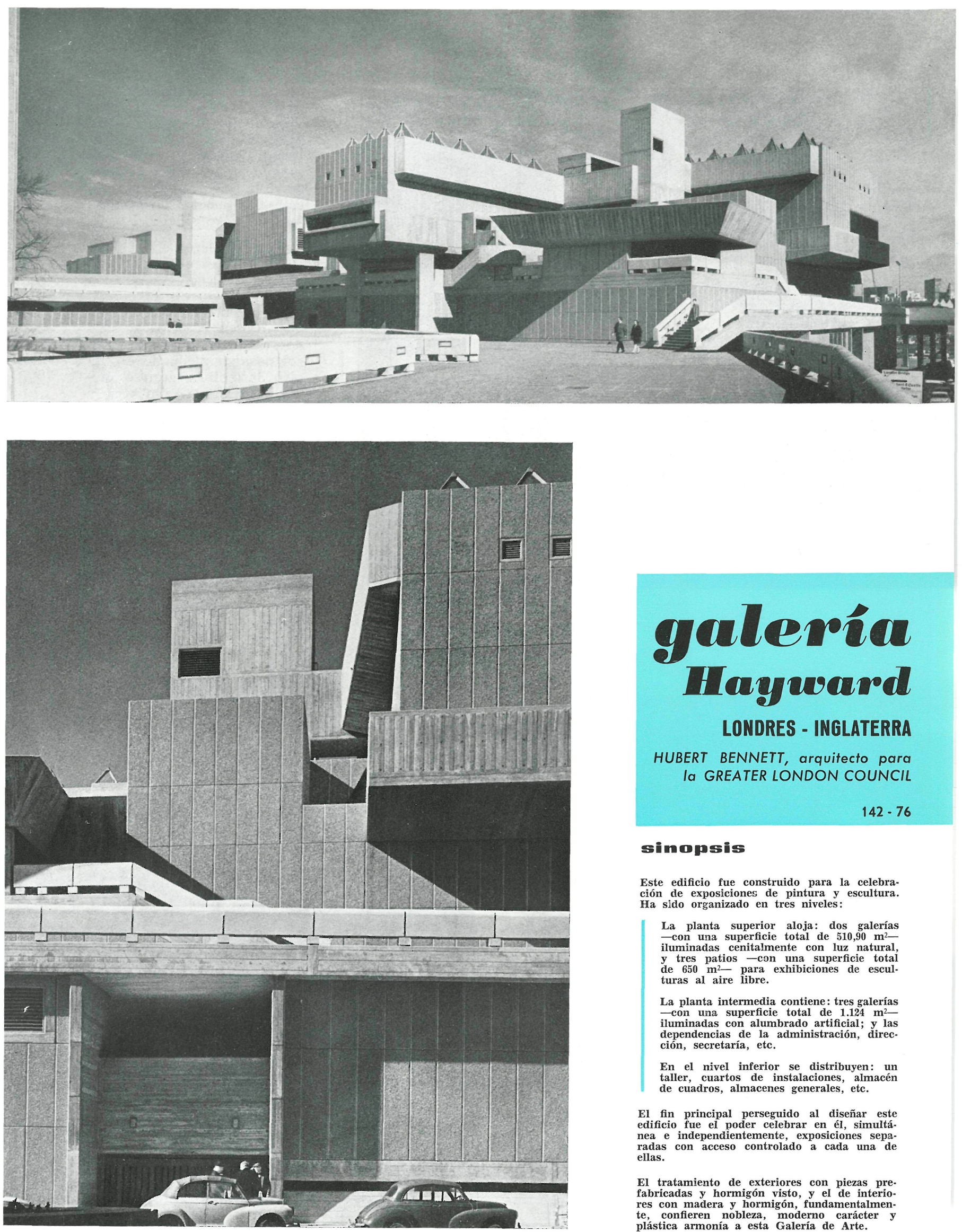

\title{
sinopsis
}

Este edificio fue construido para la celebración de exposiciones de pintura y escultura. Ha sido organizado en tres niveles:

La planta superior aloja: dos galerías - con una superficie total de $510,90 \mathrm{~m}^{2}$ iluminadas cenitalmente con luz natural, y tres patios -con una superficie total
de $650 \mathrm{~m}^{2}$ para exhibiciones de esculturas al aire libre.

La planta intermedia contiene: tres galerías -con una superficie total de $1.124 \mathrm{~m}^{2}-$ iluminadas con alumbrado artificial; y las ción, secretaría, etc.

En el nivel inferior se distribuyen: un taller, cuartos de instalaciones, almacén

E1 fin principal perseguido al diseñar este edificio fue el poder celebrar en él, simultánea e independientemente, exposiciones sepa-
radas con acceso controlado a cada una de ellas.

El tratamiento de exteriores con piezas prefabricadas y hormigón visto, $y$ el de interiores con madera y hormigon, fundamentalmenplástica armonía a esta Galería de Arte. 


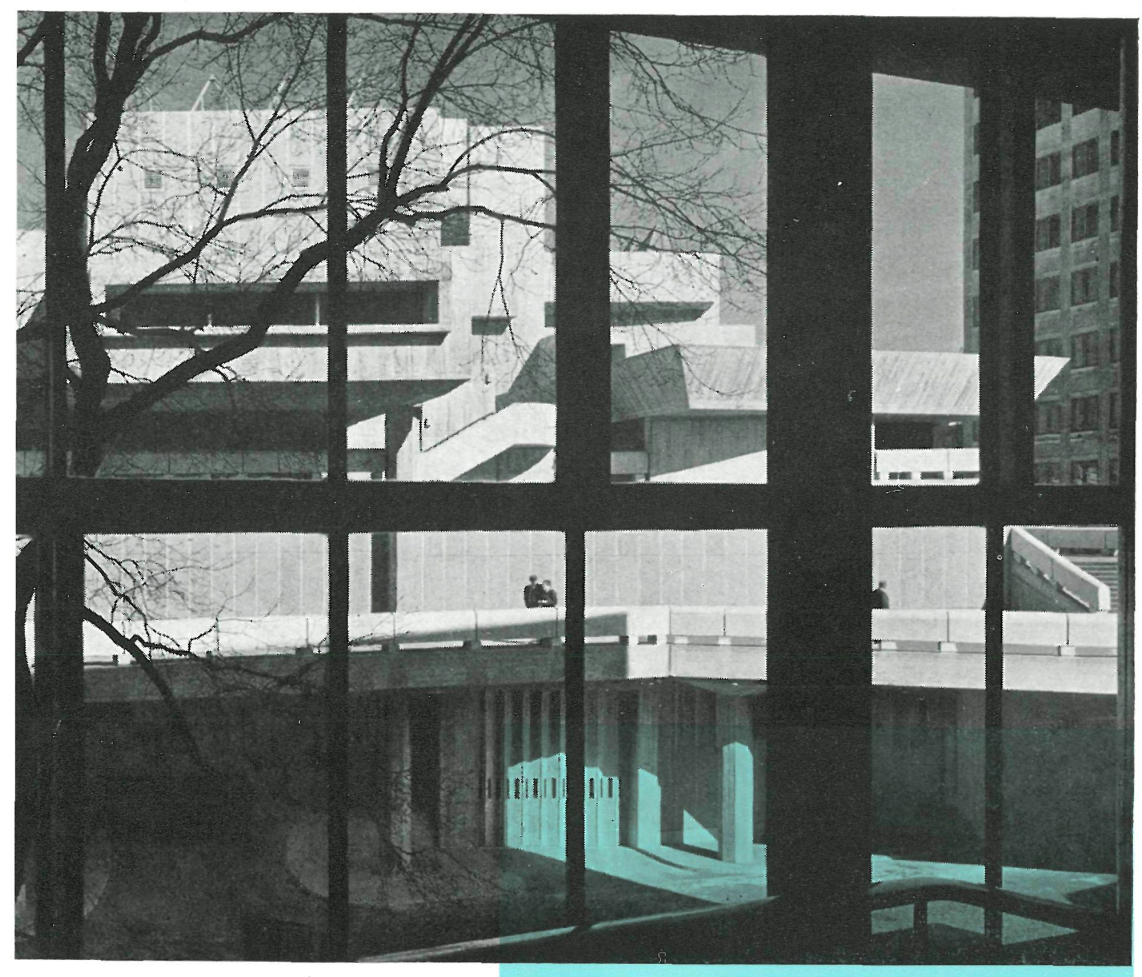

Con la inauguración de la Galería de Arte Hayward ha sido completado el conjunto de edificios que constituyen el "South Bank Arts Centre», que es un complejo artístico situado en la orilla S. del Támesis, entre los puentes de Hungerford y de Waterloo.

En esta Galería se exhiben cuadros y esculturas y fue proyectada a la vez que los edificios "Queen Elizabeth Hall» y «Purcell Room»; estos dos últimos edificios, unificados desde el punto de vista compositivo mediante el empleo de motivos arquitectónicos y materiales similares, y relacionados con el Royal Festival Hall por medio de un sistema de pasos - en terraza- para peatones, que facilitan el acceso desde la confluencia de la estación de Waterloo con los puentes de Waterloo y Hungerford.

El acceso principal de peatones a la Galería se realiza también desde estos pasos en
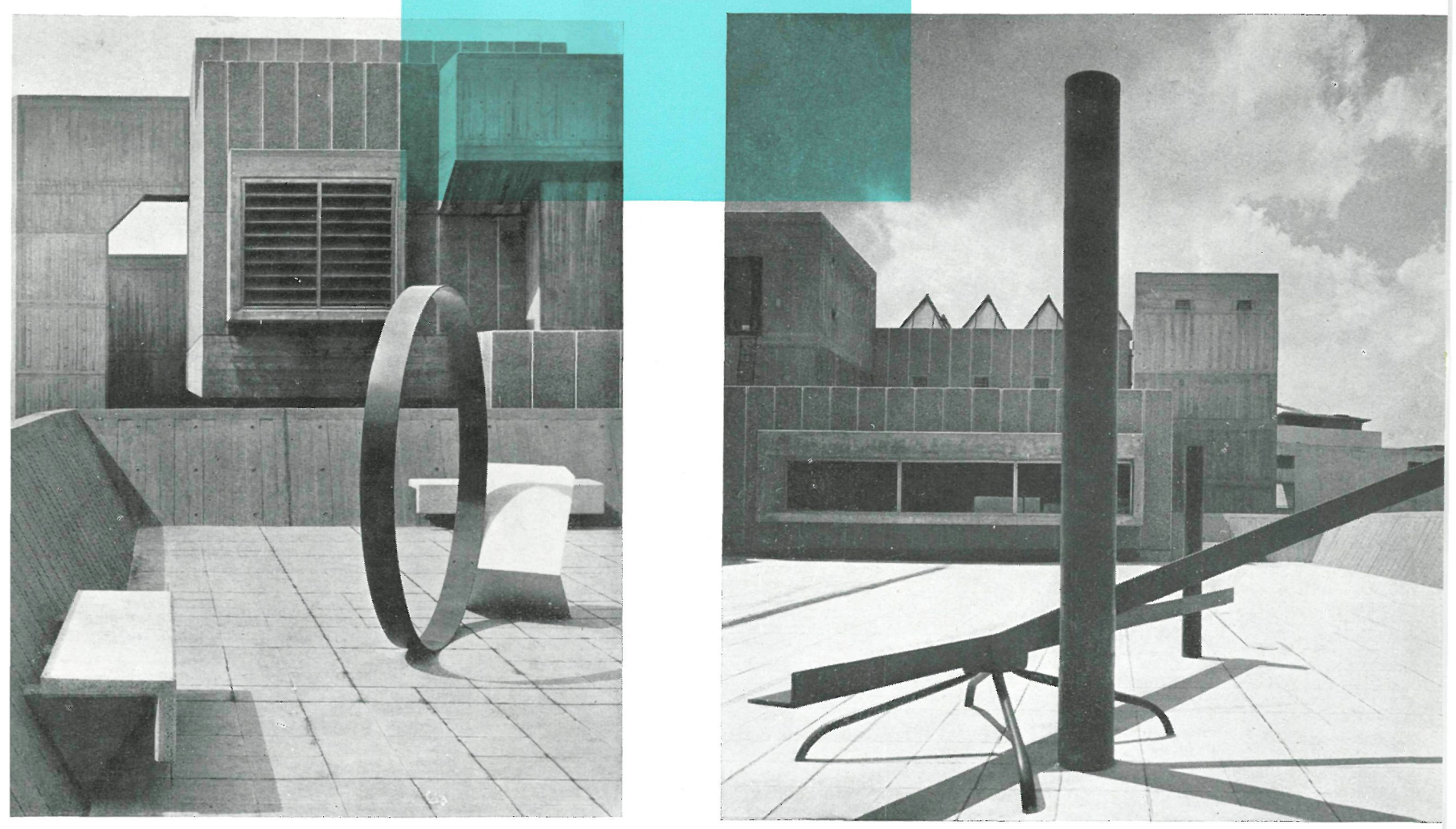


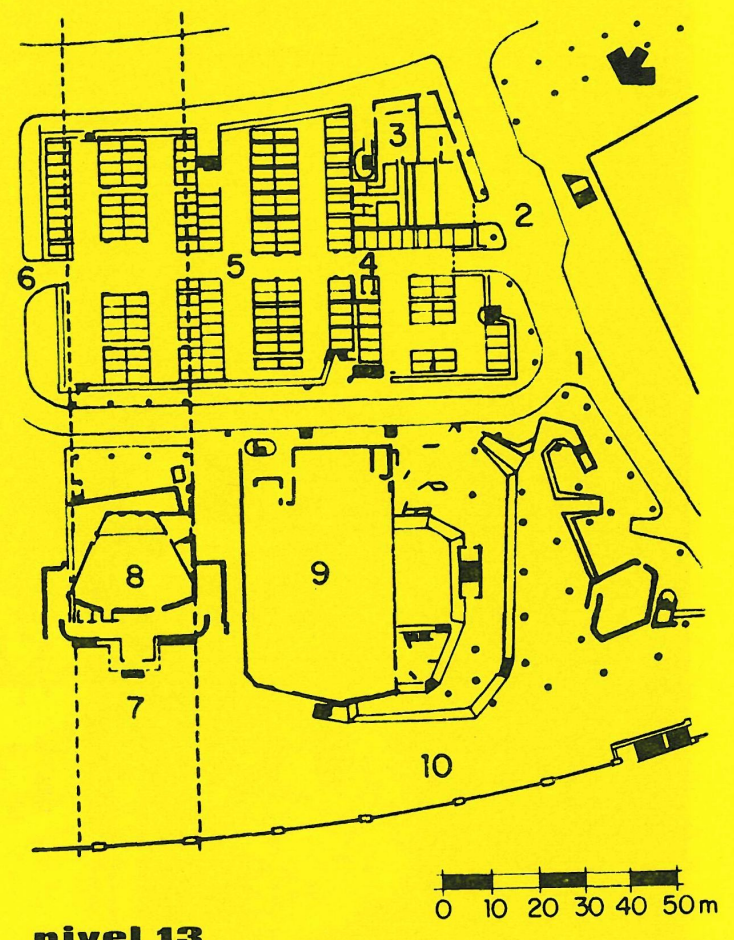

pat a ta

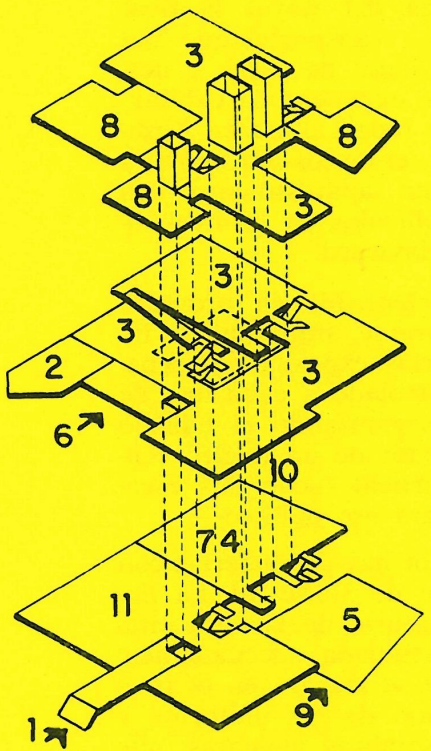

esquema

alesplazudo

1. Entrada de coches.

ria. - 4. Ascensor. -

5. Andén de carga.6. Entrada de peato nes. -7. Sala de utilla je.-8. Patio de escul10. Conductos de servicios.-11. Almacén.

\section{mivel 13}

1. Carretera de acceso.-2. Entrada al andén de carga.-3. Almacén, sala de utillaje.-4. Ascensor a la galería.-5. Aparcamiento.-6. Entrada al aparcamiento. - 7. Entrada a N.F.T.Purcell.-10. Paseo al lado del río.
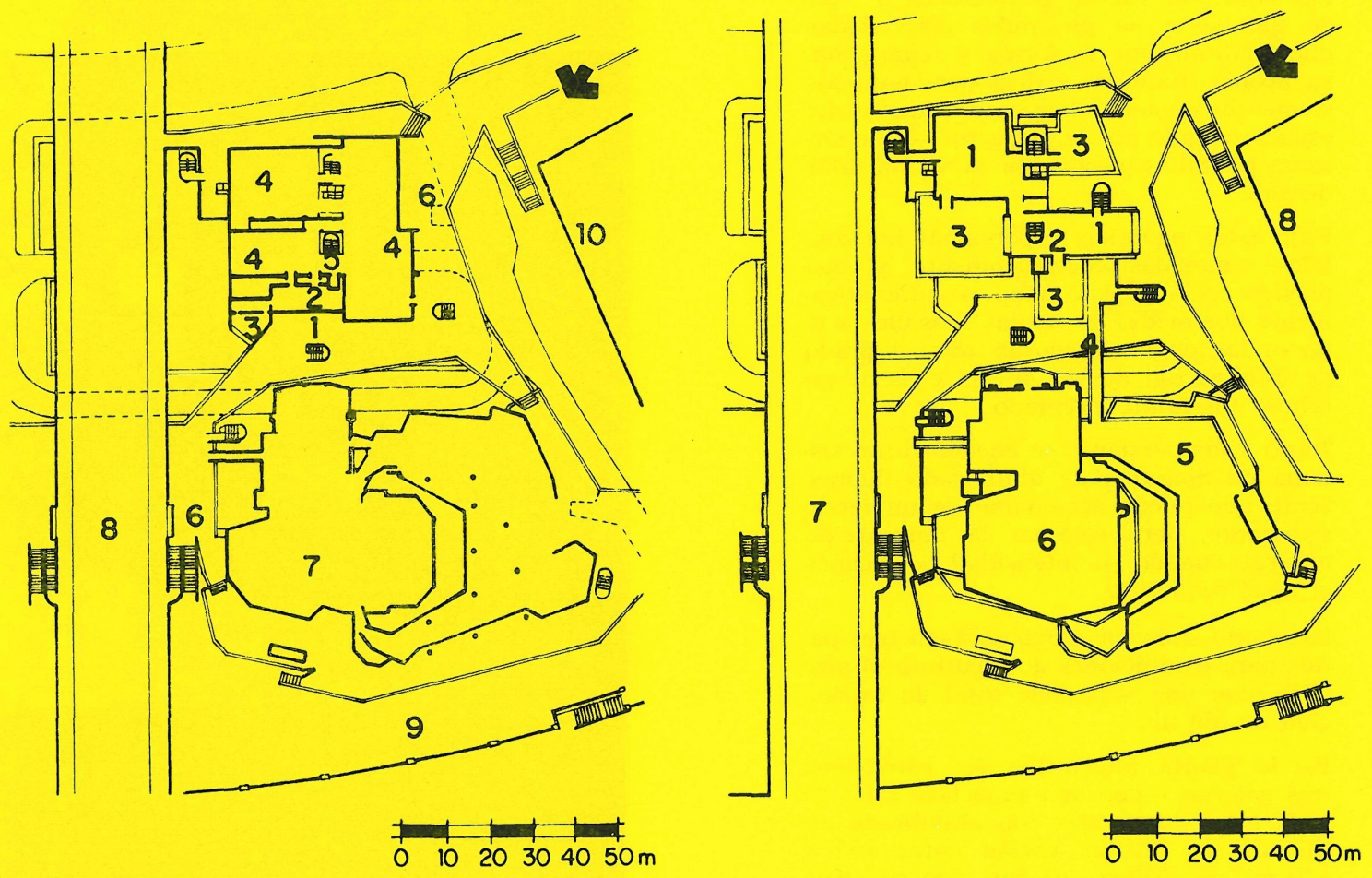

\section{mivel 32}

1. Entrada principal a la galería.-2. Vestíbulo.-3. Director.4. Galería.-5. Ascensor.-6. Terraza de peatones.-7. Sala de 9. Paseo al lado del río.-10. Sala del Royal Festival.

\section{mivel 57}

1. Galeria.-2. Ascensor.-3. Patio de esculturas.-4. Puente. 5. Terraza de peatones. -6 . Sala de Queen Elizabeth y Salón Purcell.-7. Puente de Waterloo--8. Sala del Royal Festival. 
terraza, y el acceso de vehículos se lleva a cabo: por la carretera del Royal Festival Hall, el de los vehículos transportes que han de verificar las operaciones de carga y descarga de los objetos a exponer o a exhibir; y por la carretera que rodea al Queen Elizabeth Hall y la Galería, el de los automóviles particulares. Existe un aparcamiento con capacidad para 150 vehículos en el nivel inferior de la Galería Hayward.

La exigencia básica planteada en proyecto de que pudieran celebrarse, simultánea e independientemente, varias exposiciones separadas, con acceso controlado a cada una de ellas, fue satisfecha organizando el edificio en tres niveles, alrededor de un núcleo central de circulación vertical - con dos escaleras y un ascensor para uso público-:

En la planta superior hay dos galerías con una superficie total de $510,90 \mathrm{~m}^{2}$. La iluminación cenital natural de las dos citadas galerías fue estudiada adecuadamente, de manera: que se lograra en el interior una distribución de luz uniforme y no se produjeran sombras violentas; que pudiera controlarse su intensidad; que no entrase luz solar directa en las galerías, y que la radiación ultravioleta quedara reducida a un nivel aceptable (esta última condición era necesaria para la conservación de los cuadros).

Las cubiertas fueron proyectadas a base de una serie de pirámides acristaladas que facilitan, por su forma y separación, su propia limpieza, y disponen, bajo los elementos de acristalamiento y dentro del espacio creado por la estructura, de oportunas pantallas para evitar la acción solar directa.

Bajo estas pantallas y persianas horizontales - movidas mecánicamente y que pueden accionarse a mano, o automáticamente por medio de células sensibles a la luz-, las cuales aseguran que el grado de iluminación dentro de las galerías no rebase un límite prefijado.

Junto a las persianas se encuentra un sistema de aparatos de alumbrado fluorescente que puede accionarse de un modo semejante, para reforzar la iluminación natural, cuando su intensidad no alcance el nivel requerido.

La planta superior aloja también tres patios para exhibiciones de escultura al aire libre, con una superficie total de exposición de $650 \mathrm{~m}^{2}$.

En la planta intermedia se distribuyen tres galerías - con una superficie total de $1.124 \mathrm{~m}^{2}$ - iluminadas con alumbrado artificial; sus alturas varían entre 3,35 y $6,10 \mathrm{~m}$; sus cielorrasos han sido construidos a base de planchas de aluminio, en las que se alojan los difusores de aire y los accesorios de las lámparas de tungs-
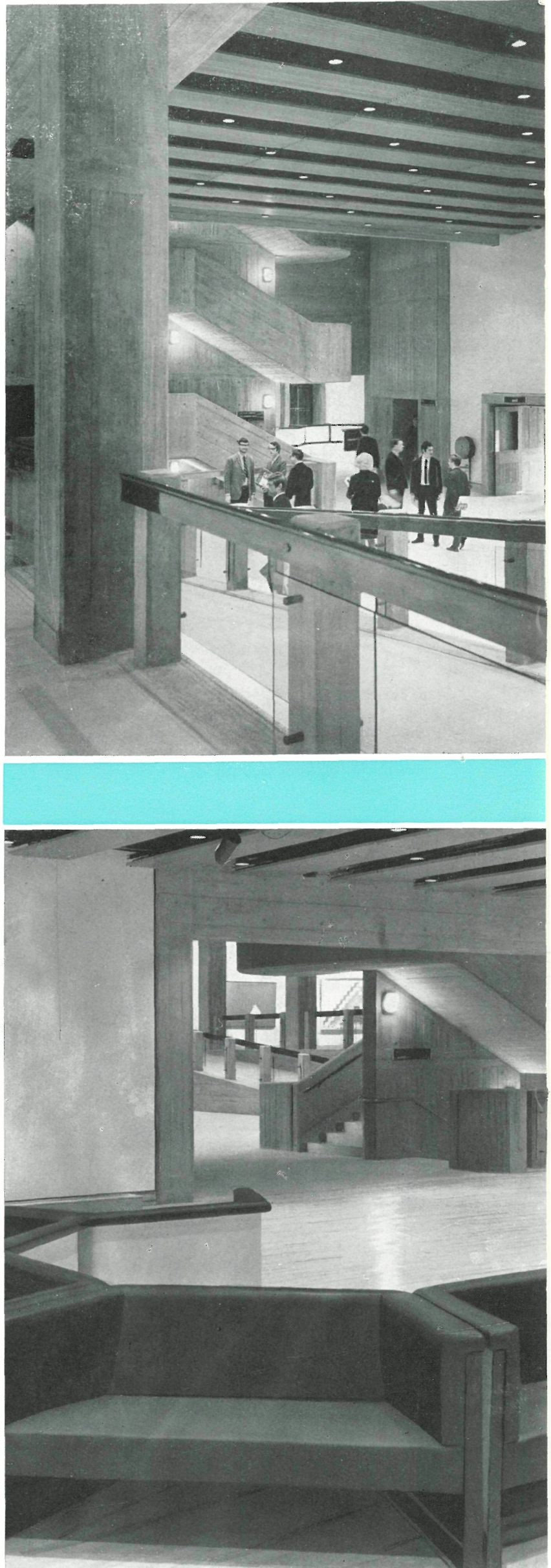
teno; asimismo, por el interior del cielorraso discurre el tendido de cables para el alumbrado, que ofrece la posibilidad de poder cambiar la posición de los puntos de luz a voluntad y adaptarlo a cualquier tipo de exposición.

Desde esta zona de las dependencias de la administración —salón, y despachos del director y secretario- se puede acceder directamente a las galerías situadas en el nivel bajo; y por medio del ascensor, a las del nivel superior.

En la planta inferior del edificio están localizados: un taller; los cuartos de las instalaciones (aire acondicionado, etc.); almacenes; aseos; y el almacén de cuadros, que está equipado con dispositivos regulables para el almacenamiento y dispone de acceso directo a un muelle de carga. Desde aquí, un elevador para objetos voluminosos transporta aquellos que se van a exponer, incluso las esculturas, a las galerías situadas en los niveles superiores.

Se ha procurado que en todas las galerías puedan celebrarse exposiciones murales, y, además, cuatro de ellas han sido equipadas con un sistema de elementos desmontables, con los que se pueden modular los espacios según un módulo de 1,22 metros.

Los paramentos de todas las galerías han sido revocados y acabados como una superficie mate; y las cajas de escaleras y ascensor comprendidas en el núcleo de circulación del edificio, manifiestan en sus paramentos la estructura de hormigón.

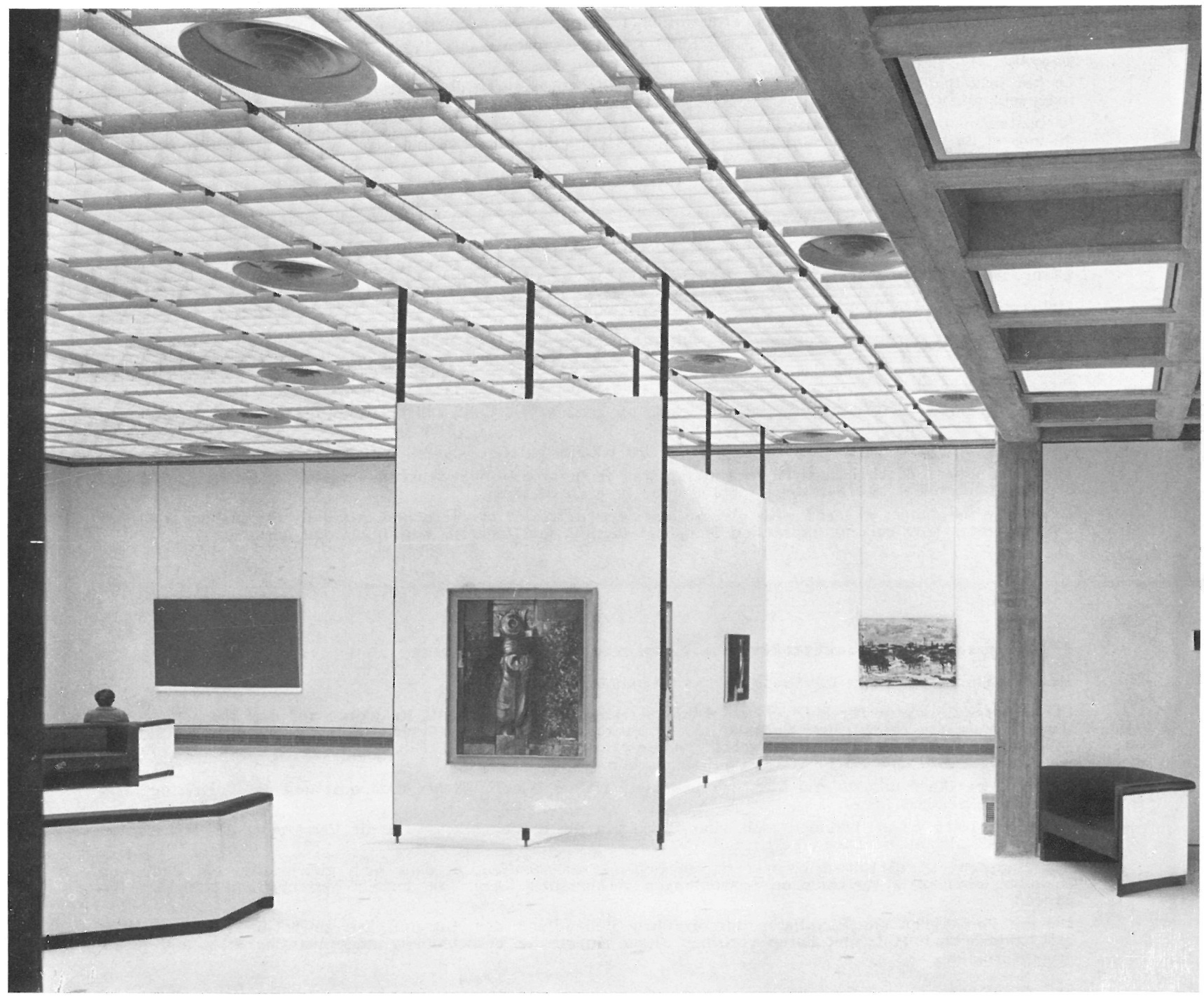


Respecto a los pavimentos: los de las cuatro galerías en las que pueden exponerse esculturas pesadas o sobre los que tales esculturas son transportadas hacia los patios exteriores, han sido realizados a base de baldosas; y el de las restantes, a base de madera.

En cuanto a la composición de los alzados exteriores - que armonizan con los del Concert Hallacusan la disposición de las galerías superiores situadas transversalmente sobre las inferiores, lo que constituye el rasgo dominante del conjunto.

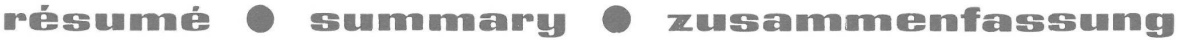

\section{Galerie Hayward di Londres - Grande-Bretagne}

Hubert Bennett, architecte au Greater London Council.

Cet édifice a été construit pour la célébration d'expositions de peinture et de sculpture. Il comprend trois niveaux:

Le niveau supérieur abrite deux galeries - dont la superficie totale est de 510,90 $\mathrm{m}^{2}$ - éclairées zénithalement par lumière naturelle, et trois cours - dont la superficie totale est de $650 \mathrm{~m}^{2}-$ pour des expositions de sculp-
tures en plein air.

Le niveau intermédiaire comprend trois galeries, ayant une superficie totale de $1.124 \mathrm{~m}^{2}$, éclairées par lumière artificielle, et les locaux de l'administration, la direction, le secrétariat, etc. Au niveau inférieur sont distribués l'atelier, les salles des installations, l'entrepôt des tableaux, les magasins
généraux, etc.

Le but principal poursuivi dans la conception de cet édifice a été de pouvoir y célébrer, simultanément et indépendamment, des expositions séparées avec accès contrôlé à chacune d'elles.

Le traitement des extérieurs avec des éléments préfabriqués et du béton apparent, et celui des intérieurs avec du bois et du béton fondamentalement confèrent un caractère noble et moderne et une harmonie plastique a cette Galerie d'Art.

\section{Haguarad Gallery, GH. Britain}

Hubert Bennett, architect to the Greater London Council.

This building is meant for painting and sculpture exhibitions. It is planned on three levels.

The top floor has two galleries, with total area of $511 \mathrm{~m}^{2}$, and is iluminated with zenithal natural light, and there are three patios, with an area of $650 \mathrm{~m}^{2}$, to exhibit sculptures in the open air.

The intermediate floor has three galleries, with an area of $1124 \mathrm{~m}^{2}$ and artificial lighting. There are also offices for the management and the secretary.

On the ground level there is a workshop, service rooms, painting stores, and general stores.

The main object which influenced this design was to be able to hold separate exhibitions simultaneously and provide independent and controlled public entry to each of them.

Externally the gallery is faced with precast units and untreated concrete, and internally the surface is timber and concrete. The general impression is one of dignity, and modern, well integrated harmony.

\section{Hegumared Kumstholle in Londom - England}

Hubert Bennett, Architekt für Greater London Council.

Dieses Gebäude wurde für Malerei- und Bildhauereiansstellungen erstellt. Es wurde auf drei Ebenen gebaut: Das Obergeschoss enthält zwei Gallerien, die durch die Decke eine natürliche Belichtung erhalten, und die eine Gesamtfläche von 510,90 qm ergeben. Ferner drei Aussenhöfe für Bildhauereiausstellungen im Freien, welche zusammen eine Fläche von $650 \mathrm{qm}$ ausmachen.

Die mittlere Etage enthält drei kürstlich belichtete Hallen (Gesamtfläche: 1.124 qm) und die Verwaltung (Direktion, Sekretariat, usw).

In der unterer Etage befinden sich eine Werkstatt, die Räumlichkeiten für die Versorgung des Hauses, Gemäldelager, allgemeine Lager, usw.

Der Hauptzweck des Entwurfes war es, ein Gebäude zu schaffen, in dem man gleichzeitig und doch von einander unabhängig verschiedene Ausstellungen veranstalten kann, mit jeweils getrennt kontrollierten Zugängen.

Die mit Fertigtielen und Sichtbeton durchgeführte Behandlung der Aussenflächen sowie die Innengestaltung aus hauptsächlich $\mathrm{Holz}$ und Beton verleihen dieser Kunsthalle Vernehmheit, modernen Charakter und plastische Harmonie. 